\title{
Structure, disorder and function of supramolecular polymer additives
}

$\underline{\text { Juergen Senker }}^{1}$, Christoph Zehe ${ }^{1}$, Marko Schmidt ${ }^{1}$, Joshua Hill ${ }^{1}$, Renée Siegel ${ }^{1}$, Venita Daebel ${ }^{2}$, Klaus Kreger ${ }^{3}$, Andrew Goodwin $^{4}$, Hans-Werner Schmidt ${ }^{3}$

${ }^{1}$ Inorganic Chemistry III, Bayreuth, Germany, ${ }^{2}$ Bruker Biospin, Karlsruhe, Germany, ${ }^{3}$ Macromolecular Chemistry I, University of Bayreuth, Bayreuth, Germany, ${ }^{4}$ Inorganic Chemistry, University of Oxford, Oxford, United Kingdom E-mail: juergen.senker@uni-bayreuth.de

This presentation intends to overview our recent progress on selected benzene trisamides (BTAs), which are powerful supramolecular polymer additives for isotactic polypropylene [1]. We could develop a basic understanding for the complex interplay between the molecular structure, the hierarchical self-asembly of the BTAs to nanoparticles and the final function of the nano-objects within the additive/polymer composites [2]. For this we made use of ab initio structure determination using the combination of powder X-ray diffraction, total X-ray scattering, computational chemistry and solid-state NMR spectroscopy. Such a joint approach helps to overcome the severe loss of information from which diffraction techniques suffer when dealing with poorly crystalline powders. In particular, by exploiting high-resolution $1 \mathrm{H}, 13 \mathrm{C}$, 15N and $19 \mathrm{~F} 1 \mathrm{D}$ spectra as well as $13 \mathrm{C} 13 \mathrm{C}$ and $19 \mathrm{~F} 19 \mathrm{~F}$ double quantum (DQ) experiments, we could guide the structure solution process of the powder diffraction data starting from the space group selection over the generation of models for the average structures up to the final refinement [1]. The combination with total X-ray scattering, furthermore, gives access to order phenomena on the mesoscale and is thus an ideal supplement for classic NMR crystallographic approaches [3].

In detail, we show that the molecular design of the BTAs needs to induce a strongly anisotropic crystal growth based on the formation of a strong and directed intermolecular $\mathrm{H}$-bond network. In polymer melts this may lead to rod-like nanoaggregates, which are supposed to serve as nucleation seeds for the crystallisation of the polymer by epitaxial lattice matching [2]. Our studies reveal, that a surprisingly rich polymorphism, the formation of macrodipoles and the assembly of the latter into mesocale domains are key issues, which have to be controlled for a successful application as polymer additive [3].

[1] Zehe, C. S. \& Schmidt,M. \& Siegel, R. \& Kreger, K. \& Daebel, V. \& Ganzleben, S. \& Schmidt, H.-W. \& Senker, J. 2014. CrystEngComm, 16, 9273.

[2] Schmidt, M. \& Wittmann, J. \& Kress, R. \& Schmidt, H.-W. \& Senker, J. 2013. Chem. Comm. 49, 267.

[3] Zehe, C. S. \& Hill, J. A. \& Funnell, N. P. \& Kreger, K. \& van der Zwan, K. P. \& Goodwin, A. L. \& Schmidt, H.-W. \& Senker, J. 2017. Angew. Chemie Int. Ed., 56, 4432.

Keywords: NMR crystallography, DQ NMR, X-ray scattering 